\title{
Breast cancer risk in women treated with augmentation mammoplasty (Review)
}

\author{
ALESSIO STIVALA $^{1}$, MASSIMO LIBRA ${ }^{2}$, FRANCA STIVALA $^{2}$ and ROSARIO PERROTTA ${ }^{1}$ \\ ${ }^{1}$ Department of Medical and Surgery Specialties, Section of Plastic Surgery, Cannizzaro Hospital, Catania; \\ ${ }^{2}$ Department of Bio-Medical Sciences, Section of Pathology and Oncology, University of Catania, Catania, Italy
}

Received November 21, 2011; Accepted January 9, 2012

DOI: $10.3892 /$ or.2012.1762

\begin{abstract}
Surgical implantation of breast prostheses has become increasingly popular while the incidence of breast cancer is increasing each year. There has been no definitive consensus regarding the casual relationship between augmentation mammoplasty and breast cancer incidence, detection, treatment, mortality and survival. This review summarizes the published evidence, including epidemiological studies and case reports. All studies examined state that there is no breast cancer risk in prior augmented women. Moreover, there is also no significant difference in frequency, stage or mean tumor size between augmented and non-augmented women.
\end{abstract}

\section{Contents}

1. Introduction

2. Risk estimate of breast cancer in women with prior breast enlargement

3. Breast cancer characteristics between women treated with prior breast implants and not treated women

4. Conclusions

\section{Introduction}

Breast cancer is by far the most frequent cancer among women with an estimated 1.38 million new cancer cases diagnosed in 2008 (23\% of all cancers), and ranks second overall (10.9\% of all cancers). It is now the most common cancer both in developed and developing regions with around 690,000 new cases estimated in each region (population ratio 1:4). Incidence rates vary from 19.3 per 100,000 women in Eastern Africa to 89.7

Correspondence to: Dr Alessio Stivala, Department of Medical and Surgery Specialties, Section of Plastic Surgery, University of Catania Cannizzaro Hospital, Via Messina 829, 95126 Catania, Italy E-mail: alessiostivala@tiscali.it

Key words: augmentation mammoplasty, breast cancer, mammography, capsule contracture, conserving surgery per 100,000 women in Western Europe, and are high (greater than 80 per 100,000) in developed regions of the world (except Japan) and low (less than 40 per 100,000) in most of the developing regions. The range of mortality rates is much less (6-19 per 100,000) because of the more favorable survival of breast cancer in (high-incidence) developed regions. As a result, breast cancer ranks as the fifth cause of death from cancer overall $(458,000$ deaths), but it is still the most frequent cause of cancer death in women in both developing $(269,000$ deaths, $12.7 \%$ of total) and developed regions, where the estimated 189,000 deaths is almost equal to the estimated number of deaths from lung cancer (188,000 deaths) (1).

Many of the established risk factors are linked to estrogens. Risk is increased by early menarche, late menopause, and obesity in postmenopausal women, and prospective studies have shown that high concentrations of endogenous estradiol are associated with an increase in risk.

Breast augmentation surgery is one of the most popular cosmetic procedures for women, annually more than 318,123 operations are performed (2). In 2011, an estimated 230,480 new cases of invasive breast cancer were expected to be diagnosed in women in the USA (30\%), along with 57,650 new cases of non-invasive (in situ) breast cancer (3). Considering these data it may be assumed that over 87,643 augmented women could develop breast cancer each year. It is therefore important to understand the potential effect of implants on breast cancer focusing on risk estimate and comparing differences between augmented and non-augmented women.

\section{Risk estimate of breast cancer in women with prior breast enlargement}

There has been no definitive consensus regarding the causal relationship between breast augmentation and breast cancer. The published data regarding breast cancer following augmentation mammoplasty are limited and contradictory.

Several cohort studies have been analysed and compared with the purpose to find a relationship between expected cases (using general population rates from the Surveillance, Epidemiology and End Results program) and effective cases (Table I).

The earliest cohort study was conducted in Los Angeles examining 3,111 cosmetic breast implant patients from the records of the private practices of 35 board-certified plastic 
Table I. Summary of cohort studies of the incidence of breast cancer in patients with augmented breasts.

\begin{tabular}{lcccc}
\hline Refs. & $\begin{array}{c}\text { No. of patients } \\
\text { with implants }\end{array}$ & $\begin{array}{c}\text { No. of breast } \\
\text { cancer patients }\end{array}$ & $\begin{array}{c}\text { No. of breast cancer cases } \\
\text { expected (SEER program) }\end{array}$ & $\begin{array}{c}\text { Standardised incidence } \\
\text { ratio (SIR) }\end{array}$ \\
\hline Deapen, et al (5) & 3,111 & 9 & 15.7 & 0.57 \\
Deapen and Brody (6) & 3,112 & 21 & 31.7 & 0.66 \\
Berkel, et al (8) & 11,676 & 41 & 86.2 & 0.48 \\
Deapen, et al (7) & 3,182 & 37 & 38.29 & 0.63 \\
Brinton, et al (9) & 13,488 & 136 & 152.2 & 0.9 \\
Brisson, et al (10) & 24,558 & 676 & 899 & 0.57 \\
Deapen (4) & 3,139 & 43 & 62.6 & 0.69 \\
\hline
\end{tabular}

A

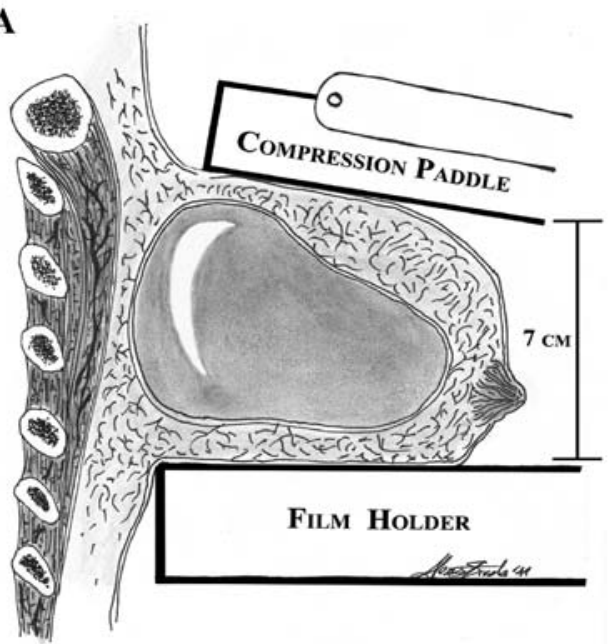

B

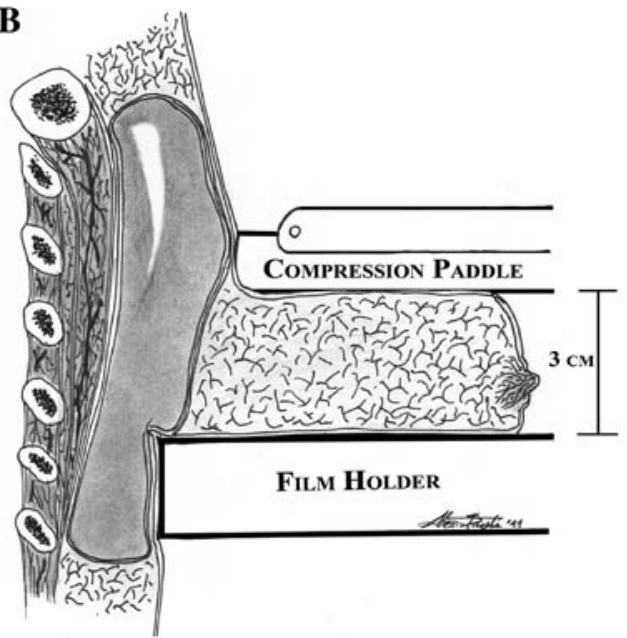

Figure 1. Mammography procedure in women treated with breast implants. (A) Mammography without Eklund technique; (B) mammography with Eklund technique.

surgeons in Los Angeles County, CA. Breast cancer diagnosis were determined by linkage with the Los Angeles Cancer Registry. The expected number of breast cancers was calculated from the population-based breast cancer rates for Los Angeles. A standardised incidence ratio was computed as the number of observed breast cancer events divided by the expected number of events. A SIR greater than one indicates the breast cancer rate in the study group exceeds that expected in the SEER area, while a SIR less than one indicates a deficit in the breast cancer rate in the study population compared to that expected. With an average of 6.2 years after implantation only $57 \%$ of the expected number of breast cancer was observed $(4,5)$. The same cohort population has been monitored and updated for more than 15 years after implantation showing a constant low cancer risk at 10.6 years $(66 \%), 14.4$ years $(63 \%)$ and at 15.5 years $(69 \%)(4,6,7)$.

Berkel et al conducted a similar study in Alberta, Canada examining a cohort of 11,676 patients with breast augmentation created from provincial medical records (8). Only $48 \%$ of the expected number of breast cancer was observed after 10.2 postimplant years.

Another important retrospective cohort study examined patients from 18 plastic surgery practices in six geographical areas (Atlanta, GA; Birmingham, AL; Charlotte, NC; Miami and Orlando, FL; and Washington, DC). Medical records of 13,488 subjects composed of all augmentation mammoplasty patients were identified for study. Cancer events were identified through questionnaires or death certificates. A total of 136 breast cancers were observed and compared with the 152.2 cases expected revealing a not statistically significant difference in breast cancer incidence (SIR 0.9) (9).

Among 24,558 cosmetic breast implant patients in the Canadian provinces of Ontario and Quebec, 676 subjects were affected of breast cancer during an average follow-up of 15.4 years (for the Quebec patients) and 13.8 years (for the Ontario patients). Data from the Canadian Cancer Registry were compared with the affected population providing a nonsignifically reduced estimate of breast cancer incidence (10).

\section{Breast cancer characteristics between women treated with prior breast implants and not treated women}

Determining breast cancer risk is not sufficient for studying the relationship between breast cancer and augmentation mammoplasty. It is therefore important to value and compare breast cancer in augmented and non-augmented patients with the purpose to establish how much a breast implant is able to affect detection, size and stage (Table II). 
Table II. Summary of the studies comparing breast cancer patients with and without implants.

\begin{tabular}{|c|c|c|c|}
\hline Study & No. of breast cancer patients & $\begin{array}{c}\text { No. of breast cancer patients } \\
\text { with implants }\end{array}$ & $\begin{array}{l}\text { No. of breast cancer patients } \\
\text { without implants }\end{array}$ \\
\hline Birdsell, et al (15) & 13,287 & 41 & 13,246 \\
\hline Clark, et al (16) & 1,768 & 33 & 1,735 \\
\hline Cahan, et al (17) & 633 & 22 & 611 \\
\hline Skinner, et al (18) & 2,956 & 99 & 2,857 \\
\hline James, et al (19) & 4,186 & 76 & 4,110 \\
\hline Tuli, et al (20) & 3,565 & 12 & 3,553 \\
\hline Handel (11) & 4,082 & 129 & 3,953 \\
\hline
\end{tabular}

Table III. Comparison of tumor stage and mean tumor size between augmented and non-augmented breast cancer patients.

\begin{tabular}{|c|c|c|c|}
\hline Study & Ductal carcinoma in situ (\%) & Invasive carcinoma $(\%)$ & Mean tumor size \\
\hline \multicolumn{4}{|l|}{ Birdsell, et al (15) } \\
\hline Augmented & 12 & 88 & $66 \%$ of patients $<2 \mathrm{~cm}$ \\
\hline Non-augmented & 3.5 & 96.5 & $34 \%$ of patients $<2 \mathrm{~cm}$ \\
\hline \multicolumn{4}{|l|}{ Skinner, et al (18) } \\
\hline Augmented & 17.2 & 82.8 & $25.5 \mathrm{~mm}$ \\
\hline Non-augmented & 41.3 & 58.7 & $29.7 \mathrm{~mm}$ \\
\hline \multicolumn{4}{|l|}{ James, et al (19) } \\
\hline Augmented & 9.6 & 90.4 & $25 \%$ of patients $<1 \mathrm{~cm}$ \\
\hline Non-augmented & 12.2 & 87.8 & $23.3 \%$ of patients $<1 \mathrm{~cm}$ \\
\hline \multicolumn{4}{|l|}{ Tuli, et al (20) } \\
\hline Augmented & 17 & 83 & $17.7 \mathrm{~mm}$ \\
\hline Non-augmented & 23 & 77 & $17.9 \mathrm{~mm}$ \\
\hline \multicolumn{4}{|l|}{ Handel (11) } \\
\hline Augmented & 27.3 & 72.7 & $23.2 \mathrm{~mm}$ \\
\hline Non-augmented & 33.3 & 66.7 & $23.8 \mathrm{~mm}$ \\
\hline
\end{tabular}

It is assumed that the presence of a prosthesis in the mammary parenchyma may interfere with the physical examination (palpability) and the radiographic imaging (mammography) increasing the risk of late detection lesions. Furthermore, scarring, fat necrosis or capsule contraction (Baker grade 1-4) may appear as thickened areas or palpable lumps in association with calcium deposits that can be misinterpreted on mammography as malignant calcifications. To minimize the shadow cast produced by an implant interfering with breast tissue visualization, a displacement technique has been introduced by Eklund et al (12) with the aim to increase mammographic sensitivity (Fig. 1).

Some authors report in singular cases that cancers in augmented women usually are more advanced at the time of detection $(13,14)$, whereas others have reported no significant difference in tumor size or stage at the time of diagnosis in augmented women compared to non-augmented women.

Tanaka et al reported in 2008 a case of three invasive micropapillary carcinomas in a 64-year-old woman who underwent breast augmentation 42 years before (14). The multiple tumors were located next to the siliconomas suggesting a possible relationship between siliconomas and the onset of carcinomas.

Birdsell et al (15) examined 13,287 women with breast cancer diagnosed between 1973 and 1990 in Alberta. Among this group, 41 women underwent augmentation mammoplasty before cancer diagnosis (Table II). The study was carried out before the beginning of breast screening programmes, so we can deduce that the majority of patients were symptomatic. The authors revealed a higher incidence of in situ carcinoma in women with implants $(12 \%)$ compared to non-implanted patients $(3.5 \%)$. Tumors in $66 \%$ of augmented women were significantly smaller in diameter $(<2 \mathrm{~cm})$ compared with $34 \%$ of women without implants (Table III). No statistically significant difference in 5- and 10-year survival was identified between the groups.

Clark et al (16) compared 1,735 non-augmented breast cancer women with 33 breast cancer augmented patients (Table II). No difference in mammografically detection was 
found between the groups. However, palpable tumors were smaller in augmented breasts.

Another study conducted by Cahan et al (17) reviewed from 1977 to 1992 the cases of 22 women with breast cancer previously treated with breast augmentation mammoplasty (Table II). These patients were compared with a group of 611 women with breast cancer but without implants. This study revealed no significant difference in incidence of in situ disease and mean tumor size (tumors in augmented patients tended to be smaller than in non-augmented women).

Between 1980 and 1999, Skinner et al (18) examined 2,956 cases of breast cancer women from Los Angeles. Ninety-nine of them had prior augmented breast (Table II). A very high percentage of augmented women presented palpable cancer $(82.9 \%)$ in contrast to the non-augmented group $(63.8 \%)$ Mammography was interpreted abnormal in $66.3 \%$ of the augmented patients and $94.6 \%$ of the non-augmented patients. Women with breast prosthesis presented more often invasive tumors $(82.8 \%)$, however, there was no significant difference in mean size tumor (Table III). Furthermore, no difference in 2- and 10-year survival has been revealed between cohort patients.

In 2004 James et al (19) identified from January 1987 to February 2002 in Florida 76 breast cancers who had previously undergone breast augmentation (Table II). The authors were able to identify the method of initial presentation in 69 cases. Seventy percent of them (48 of 69) were palpable, whereas the remaining $30 \%$ (21 of 69) were mammographically identified. During the same period a cohort population of 4,110 non-augmented breast cancer patients was compared for tumor size and stage. Women with prior breast augmentation were more likely to have smaller tumors. Twenty-five percent of augmented patients ( 25 of 73 women) presented a tumor mean size $<1 \mathrm{~cm}$. This compares with $23.3 \%$ (957 of 4,110) in the control group (Table III). Even though only $9.6 \%$ of augmented patients presented with carcinoma in situ, the two groups do not differ significally in tumor stage.

An extensive study of medical records was conducted by Tuli et al (20) at the Thomas Jefferson University Hospital Department of Surgery from 1998 to 2004 examining 12 prior augmented breast patients among 3,565 breast cancer patients (Table II). Tumor detection has been achieved through selfclinical breast examination in eight cases $(67 \%)$, whereas in only four patients $(33 \%)$ breast abnormalities were visualizable by screening mammography. Even in this study there is no significant difference either in tumor mean size or in tumor stage (Table III).

In 2007, Handel et al (11) published a study comparing 129 prior augmented breast cancer patients with a control case group of 3,953 breast cancer patients treated between 1981 and 2004 at the Breast Center in Van Nuys, and at the Kenneth Norris Comprehensive Cancer Center in Los Angeles (Table II). Eighty-seven augmented and 1,741 non-augmented patients had palpable cancers. Among these groups mammography was positive in $58.6 \%$ of augmented cases and in $91.2 \%$ of non-augmented cases. Infiltrating ductal tumors were compared in size revealing no significant difference between the two groups. Compared to previous studies, in situ disease has a higher percentage frequency among the augmented cases (27.3\%) (Table III).

\section{Conclusions}

Extensive research has shown that breast implants do not increase the risk of breast cancer incidence. All current studies report a non-significant standardized incidence ratio. However, it is inevitable that many augmented women will eventually develop breast cancer. Several studies document persistent concerns about the relationship between breast implants and breast cancer detection even adopting the Eklund's technique.

Tumor palpability has been found to be a relevant characteristic among the augmented population. In non-symptomatic patients if mammography is not sufficient, magnetic resonance imaging should be considered as a valuable choice in suspected cases.

Breast cancer characteristics do not differ among the prior breast augmented patients and non-breast augmented patients. Data reveal a lower mean tumor size among the women who underwent breast enlargement compared to the control population in association with a more frequent incidence of infiltrating breast cancer.

The respect of the proper oncologic principles and the preservation of an aesthetically acceptable breast shape are the most determining factors of an effective treatment. Although breast conserving therapy may appear aesthetically satisfying, many severe implant distortions and postoperative radiotherapy complications can occur. Modified radical mastectomy with immediate reconstruction may achieve superior aesthetic results $(11,19,21,22)$.

\section{References}

1. Globocan 2008: Breast Cancer Incidence and Mortality Worldwide in 2008 Summary. IARC, Lyon, 2008.

2. American Society for Aesthetic Plastic Surgery: Statistics on Cosmetic Surgery 2010. Cosmetic Surgery Data Bank, American Society for Aesthetic Plastic Surgery Communication Office, New York, 2011.

3. American Cancer Society: Breast Cancer Facts and Figures 2011. American Cancer Society, Atlanta, 2011.

4. Deapen D: Breast implants and breast cancer: a review of incidence, detection, mortality, and survival. Plast Reconstr Surg 120 (7 Suppl 1): S70-S80, 2007.

5. Deapen DM, Pike MC, Casagrande JT and Brody Garry S: The relationship between breast cancer and augmentation mammaplasty: an epidemiologic study. Plast Reconstr Surg 77: 361-367, 1986.

6. Deapen DM and Brody GS: Augmentation mammaplasty and breast cancer: a 5-year update of the Los Angeles study. Plast Reconstr Surg 89: 660-665, 1992.

7. Deapen D, Hamilton A, Bernstein L and Brody GS: Breast cancer stage at diagnosis and survival among patients with prior breast implants. Plast Reconstr Surg 105: 535-540, 1999.

8. Berkel H, Birdsell DC and Jenkins H: Breast augmentation: a risk factor for breast cancer? N Engl J Med 326: 1649-1653, 1992.

9. Brinton LA, Lubin JH, Burich MC, et al: Breast cancer following augmentation mammoplasty (United States). Cancer Causes Cantrol 11: 819-827, 2000.

10. Brisson J, Holowaty EJ, Villeneuve PJ, Xie L, Ugnat AM, Latulippe L and Mao Y: Cancer incidence in a cohort of Ontario and Quebec women having bilateral breast augmentation. Int J Cancer 118: 2854-2862, 2006.

11. Handel N: The effect of silicone implants on the diagnosis, prognosis, and treatment of breast cancer. Plast Reconstr Surg (7 Suppl 1): S81-S93, 2007.

12. Eklund GW, Busby RC, Miller SH and Job JS: Improved imaging of the augmented breast. AJR Am J Roentgenol 151: 469-473, 1988. 
13. Kasamaki S, Tsurumaru M, Kamano T, Kobayashi S, Hino M and Kawatsuru R: A case of inflammatory breast cancer following augmentation mammoplasty with silicone gel implants. Breast Cancer 7: 71-74, 2000.

14. Tanaka Y, Moroshima I and Kikuchi K: Invasive micropapillary carcinomas arising 42 years after augmentation mammoplasty: a case report and literature review. World J Surg Oncol 6: 33, 2008.

15. Birdsell DC, Jenkins $H$ and Berkel H: Breast cancer diagnosis and survival in women with and without breast implants. Plast Reconstr Surg 92: 795-800, 1993.

16. Clark III CP, Peters GN and O'Brien KM: Cancer in the augmented breast. Diagnosis and prognosis. Cancer 72: 2170-2177, 1993.

17. Cahan AC, Ashikari R, Pressman P, Cody H, Hoffman S and Sherman JE: Breast cancer after breast augmentation with silicone implants. Ann Surg Oncol 2: 121-125, 1995.

18. Skinner KA, Silberman H, Dougherty W, et al: Breast Cancer after augmentation mammoplasty. Ann Surg Oncol 8: 138-144, 2001.
19. James W, Jakub JW, Ebert MD, Cantor A, Gardner M, Reintgen DS, Dupont EL, Cox CE and Shons AR: Breast cancer in patients with prior augmentation: presentation, stage, and lymphatic mapping. Plast Reconstr Surg 114: 1737-1742, 2004.

20. Tuli R, Flynn RA, Brill KL, Sabol JL, Usuki KY and Rosenberg AL: Diagnosis, treatment, and management of breast cancer in previously augmented women. Breast J 12: 343-348, 2006.

21. Karanas YL, Leong DS, Da Lio A, Waldron K, Watson JP, Chang $\mathrm{H}$ and Shaw WW: Surgical treatment of breast cancer in previously augmented patients. Plast Reconstr Surg 111: 1078-1086, 2003.

22. Colleen M, McCarthy CM, Pusic AL, Disa JJ, Cordeiro PG, Cody HS III and Mehrara B: Breast cancer in the previously augmented breast. Plast Reconstr Surg 119: 49-58, 2007.

23. Gray RJ, Forstner-Barthell AW, Pockaj BA, Schild SE and Halyard MY: Breast-conserving therapy and sentinel lymph node biopsy are feasible in cancer patients with previous implant breast augmentation. Ann J Surg 188: 122-125, 2004. 